\title{
Über die Dispersion der optischen Symmetrieachsen von Gips, Augit und Adular im kurzwelligen Ultrarot ${ }^{1}$ ). \\ Von Erich Goens in Berlin.
}

Mit zehn Abbildungen. (Eingegangen am 3. Mai 1921.)

In zwei Arbeiten ist von Herrn Rubens') gezeigt worden, daß die optischen Symmetrieachsen monokliner Kristalle ${ }^{3}$ ) im ultraroten Reststrahlengebiet eine erhebliche Dispersion aufweisen und sich im Einklang mit der elektromagnetischen Lichttheorie asymptotisch den dielektrischen Vorzugsrichtungen nähern. Da ferner von Herrn D ufe t: im sichtbaren Gebiet an Kristallen des Neodym- und Praseodymsulfats anomale Dispersion der Symmetrieachsen in der Nähe von Absorptionsstreifen gefunden wurde, erschien es wünschenswert, den Verlauf der Achsendrehung in dem durch prismatische Zerlegung zugänglichen Teil des ultraroten Spektrums zu untersuchen, in welchem im Gegensatz zum Reststrahlengebiet die Möglichkeit stetiger Wellenlängenänderungen bei gesteigerter Homogenität der Strahlung gegeben ist.

Versuchsanordnungen und Meßverfahren.

Die Lage der Symmetrieachsen wurde mit Hilfe eines bereits von Herrn $R$ abens verwandten Verfahrens ermittelt. Man läßt linear polarisierte monocbromatische Strahlung senkrecht auf eine parallel zur (010)-Ebene geschnittene Platte eines monoklinen Kristalls anffallen und bestimmt aus der Abhängigkeit des Durchlässigkeits- bzw. Reflexionsvermögens von der Orientierung des Kristalls gegen die Schwingungsebene des elektrischen Vektors die Azimute maximaler und minimaler Durchlässigkeit bzw. Reflexion, diese geben die Lage der beiden Symmetrieachsen für die betreffende Wellenlänge.

Die Versuchsanordnungen sind in Fig. 1 a (Durchlässigkeitsmessungen) und Fig. 1 b (Reflexionsmessungen) dargestellt. Die Strablung eines Nernstbrenners $N$ wurde durch Reflexion an einem Selenspiegel Se anter dem Polarisationswinkel polarisiert und durch ein Spiegelspektrometer $S p$ mit Wadsworthscher Anordnung s) zur

\footnotetext{
1) Gekürzte Berliner Dissertation.

2) H. Rubens, Berl. Ber. 1919, S. 976 und ZS. f. Phys. 1, 11, 1920.

3) Untersucht wurden Gips, Augit, Adular und Rohrzucker.

4) H. Dufet, Bull. soc. Franc. min. 24, 373, 1901.

5) L. Wadsworth, Phil. Mag. (5) 38, 337, 1894.
} 
Erhaltung des Minimums der Ablenkung spektral zerlegt [Prismen (birech. Winkel $60^{\circ}$ ) zwischen $1 \mu$ bis $8 \mu$ Flußspat, $8 \mu$ bis $13 \mu$ Steinsalz]. Zur Strablungsmessung diente ein Mikroradiometer $R$ in der von Herrn H. Schmidt ${ }^{1}$ ) beschriebenen Form. $H_{1}, H_{2}, H_{3}$ und $P$, $P_{1}, P_{2}, P_{3}$ sind versilberte Hohl- bzw. Planspiegel, $K$ ein Klappschirm, $M$ und $G$ Schutzgehäuse. Die Vorrichtung zum Drehen der Kristallpräparate bestand bei den Durchlässigkeitsmessungen in einer Drehscheibe $D$ von der Form eines Linsenbalters einer optischen Bank, dessen schwerer Fuß durch Anschläge (in der Fig. 1 a angedeutet) so fixiert war, daß sich der aufgebrachte Kristalldünnschliff etwas außex-

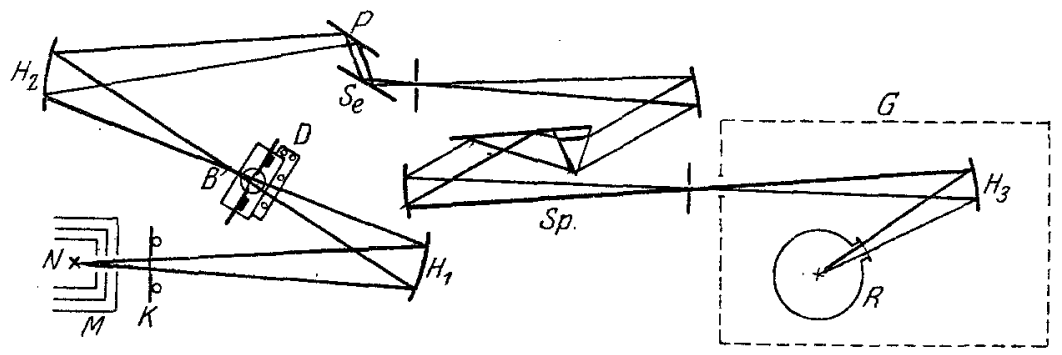

Fig. 1 a.

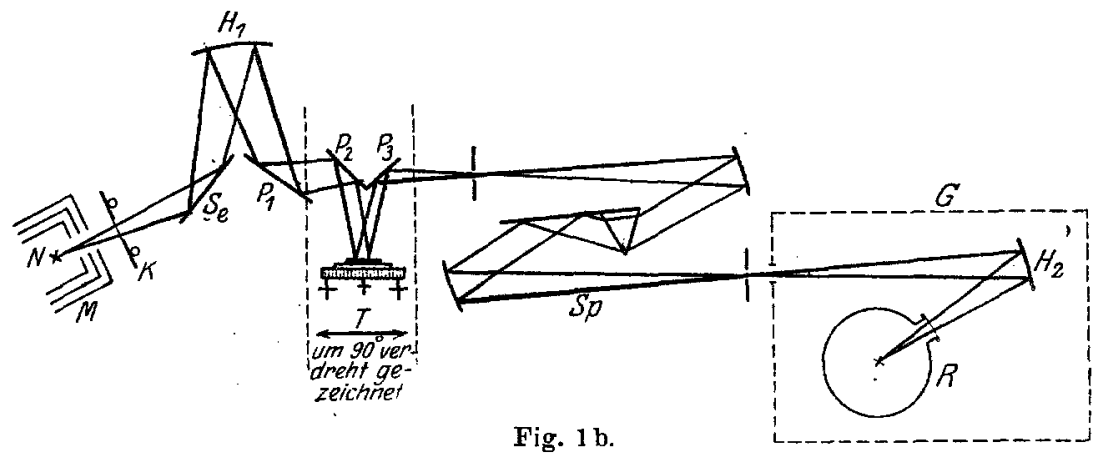

halb des Vereinigungspunktes $B$ befand. Bei den Reflexionsmessungen wurde der Kristall auf einer Unterlage anf dem Stelltisch $T$ gedreht, wobei die Parallelität der reflektierenden Fläche mit Hilfe einer Dosenlibelle aufrecht erbalten wurde.

Die Messungen vollzogen sich folgendermaßen. Die Kristallpräparate wurden mit einer äußerlich kenntlichen Vorzugsrichtung parallel zur Indexlinie auf dem drehbaren Teil der Vorrichtungen befestigt, schrittweise um $22 \mathrm{1} / \mathrm{2}^{\circ}$ gedreht und in den einzelnen Lagen das Durchlässigkeits- bzw. Reflexionsvermögen bestimmt, also bei

1) H. Schmidt, Ann. d. Phys. (4) 29, 1001, 1909. 
einer vollen Umdrehung für 16 Lagen des Kristalls. In den meisten Fällen beschränkten sich die Messungen auf eine halbe Umdrehung, da von da ab die Werte sich wiederholen müssen. Die Ansgangslage (Nullinie der Teilung) bildete fast genau die Sehwingungsebene des elektrischen Vektors. Der Drehungssinn war in der Strahlrichtung gesehen so, daß sich die Schwingungsebene des elektrischen Vektors im positiven Sinn gegen die Vorzugsrichtung drehte. Zum Zwecke der Energiemessung wurde die Drehscheibe fortgenommen bzw. die refiektierende Kristallfläche durch einen Silberspiegel ersetzt, dessen Reflexionsvermögen in dem in Frage kommenden Spektralbereich $(8 \mu$ bis $13 \mu)$ gleich 100 Proz. gesetzt wurde. Die Auftragung der so erhaltenen Durchlässigkeits- bzw. Reflexionswerte als Funktion der Drehungswinkel (wo die Werte nur zwischen $0^{\circ}$ bis $180^{\circ}$ gemessen waren, wurde ihre Auftragung wiederholt) ergeben eine sinusartige Kurve, in folgendem kurz als "Drehkurve" bezeichnet, mit zwei Maximis und Minimis, deren Winkelwerte paarweise der Lage der Symmetrieachsen bezogen auf die Vorzugsrichtung entsprachen (vgl. z. B. Fig. $4,10,2 \mu$ und $10,8 \mu$ ). Die genauen Werte wurden nach dem Vorgange von Herm Rubens ${ }^{1}$ ) durch Bestimmung der Schwerlinien in den konkaven und konvexen Kurvenbögen ermittelt.

Bei den Reflexionsmessungen war, wie aus Fig. $1 \mathrm{~b}$ ersichtlich, aus Gründen der bequemeren Aufstellung der reflektierende Kristallspiegel im Strahlengange $z$ wischen dem Polarisator Se und dem Spiegelspektrometer $\mathrm{S} p$ angeordnet. Letzteres übt gleichfalls eine schwache polarisierende Wirkung aus, wobei die Polarisationsrichtung zu derjenigen des Selenspiegels senkrecht gerichtet ist. Diese Spektrometerpolarisation kann jedoch, wie leicht zu übersehen, weder anf die beobachtete Lage der Symmetrieachsen, noch auf die Werte des maximalen und minimalen Reflexionsvermögens einen merklichen Einfluß haben; nur nehmen aus diesem Grunde die Drehkurven in einigen Fällen eine Form an, welche von der Gestalt einer Sinuskurve insofern abweicht, als die Maxima etwas zu breit, die Minima za schmal erscheinen.

Endlich wurde die geringe Abweichung der Schwingungsebene des elektrischen Vektors von der Nullinie der Teilung durch Anwendang der Verfahren auf eine achsenparallele Quarzplatte mit bekannter Lage der Vorzugsrichtangen gemessen und als Korrektionen angebracht.

Untersucht wurden mit Hilfe von Durchlässigkeitsmessungen: Gips im Bereich von $2,4 \mu$ bis $8,2 \mu$, mit Hilfe von Reflexionsmessungen:

1)'H. Rubens, 1. c. 
Gips im Bereich von $8,2 \mu$ bis $12,0 \mu$, Angit zwischen $8,6 \mu$ bis $12,8 \mu$ und Adular zwischen $8,2 \mu$ bis $10,0 \mu$. Die benutzten Kristallpräparate waren größtenteils die bereits von Herrn Rubeus verwandten. Die Resultate sind in Tab. 1 bis 3 und in Fig. 2, 5 und 6 enthalten. Erstere geben für die einzelnen Wellenlängen das Durchlässigkeitsbzw. Reflexionsvermögen für die parallel za den Achsen schwingende Strahlung ( $D_{\max }$ und $D_{\min }$, bzw. $R_{\max }$ und $R_{\min }$, in folgendem als "Hauptdurchlässigkeits-" bzw. "Hauptreflexionswerte" bezeichnet), ferner die Azimute der zugehörigen Schwingungsrichtungen $\varphi_{\max }$ und $\varphi_{\min }\left(0^{\circ} \geqq \varphi>180^{\circ}\right)$, endlich den Winkel $\varphi_{0}$ einer bestimmt gewählten Symmetrieachse, deren Bewegung mit der Wellenlänge in Fig. 2, 5 und 6 zusammen mit dem Verlauf der Hauptdurchlässigkeitsbzw. Reflexionswerte wiedergegeben ist. Die vorausgehende Angabe von $\varphi_{\max }$ und $\varphi_{\min }$ erschien angebracht, da, wie sich zeigen wird, die Wahl der Werte für $\varphi_{0}$ an gewisse, experimentell nicht sicher zu beweisende Voraussetzungen über den Verlauf der Hauptdurchlässigkeits- and Reflexionswerte geknïpft ist, während $\varphi_{\max }$ und $\varphi_{\min }$ eindeatig definiert sind.

\section{Die Ergebuisse.}

Gips $\left(\mathrm{CaSO}_{4} \cdot 2 \mathrm{H}_{2} \mathrm{O}\right)$. Die Dispersion der optischen Symmetrieachsen in Gips ist für das sichtbare Gebiet bereits mehrfach untersucht worden, zuletzt und wohl am genauesten von Her'n Berek ${ }^{1}$ ).

Die Ergebuisse der vorliegenden Messungen sind in Tab. 1 und Fig. $2 a$ and $b$ enthalten. Für die Untersuchung des vor dem ersten Streifen metallischer Reflexion $(8,7 \mu)$ liegenden Gebietes ist die Durchlässigkeitsmethode wegen des meist beträchtlichen Dichroismus am geeignetsten. Verwandt wurden zwei Spaltstücke aus dem gleichen Material (Fundort: Wimmelburg b. Eisleben), eins von $0,15_{5} \mathrm{~mm}$ Dicke im Bereich von $2,4 \mu$ bis $6,4 \mu$ und eins von $0,04_{3} \mathrm{~mm}$ im Bereich von $6,0 \mu$ bis $8,2 \mu$, deren Beschaffenheit unter dem Polarisationsapparat geprüft wurde. Sie wurden so auf die Drehscheibe gebracht, daß man in der Strahlrichtung auf die (010)-Ebene sah und die Richtang des faserigen Bruchs mit der Indexlinie zusammenfiel. Diese Richtung bildet mit der Vertikalachse einen Winkel von $66^{\circ}$.

Im Bereich des mit dem dickeren Blättchen durchmessenen Spektralgebietes zeigen die Karven für die Hauptdurchlässigkeitswerte (Fig. $2 \mathrm{~b}$ ) gemeinsam die Kristallwasserbande bei $2,9 \mu$ und die Absorptionsbande des $\mathrm{SO}_{4}$-Ions bei $4,55 \mu$. Der feinen Bande bei $2,50 \mu$ in

1) M. Berek, Neues Jahrb. f. Min., Beil., 33, 583, 1912. 


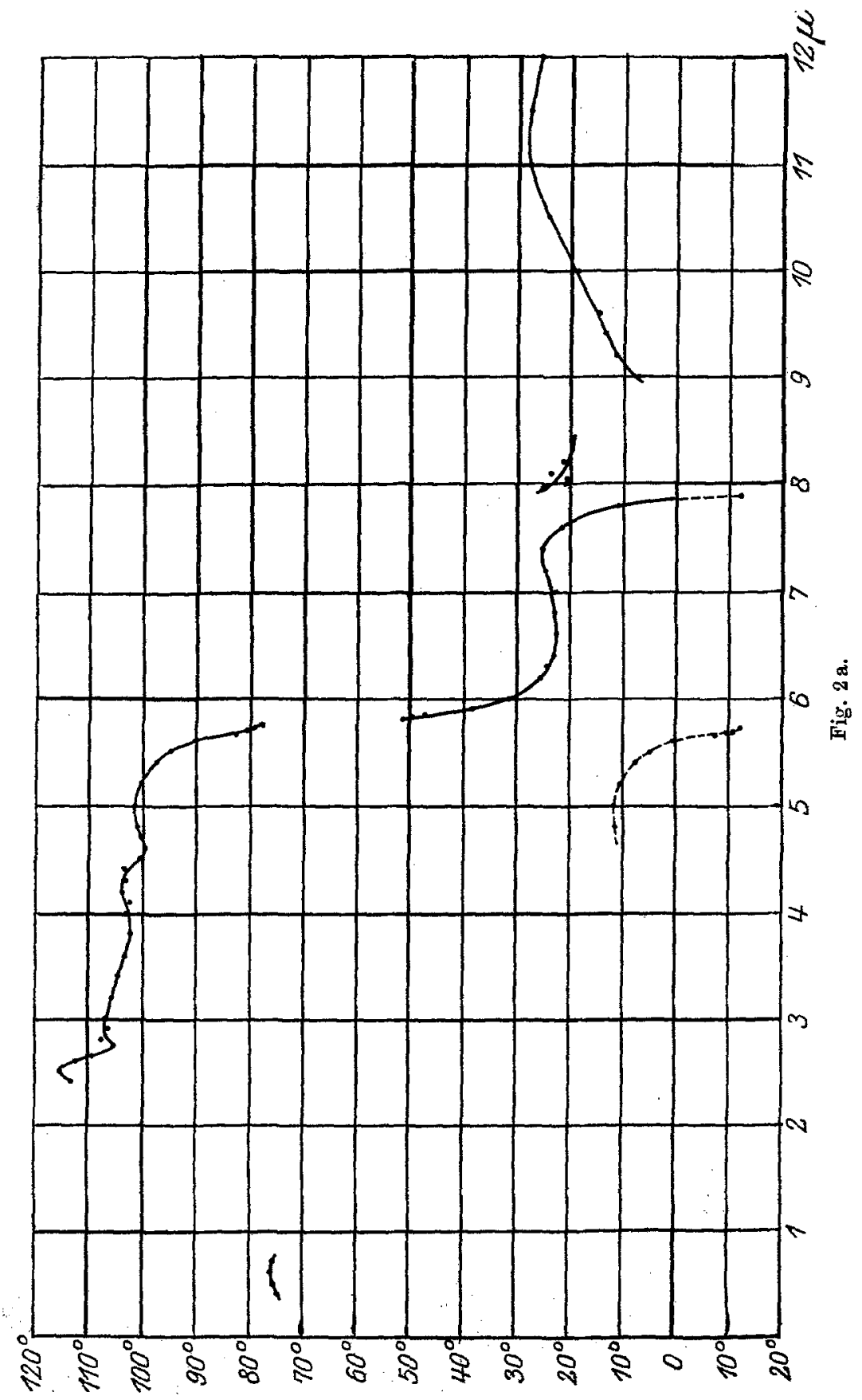


Üher die Dispersion der optischen Symmetrieachsen von Gips, Augit usw. 17

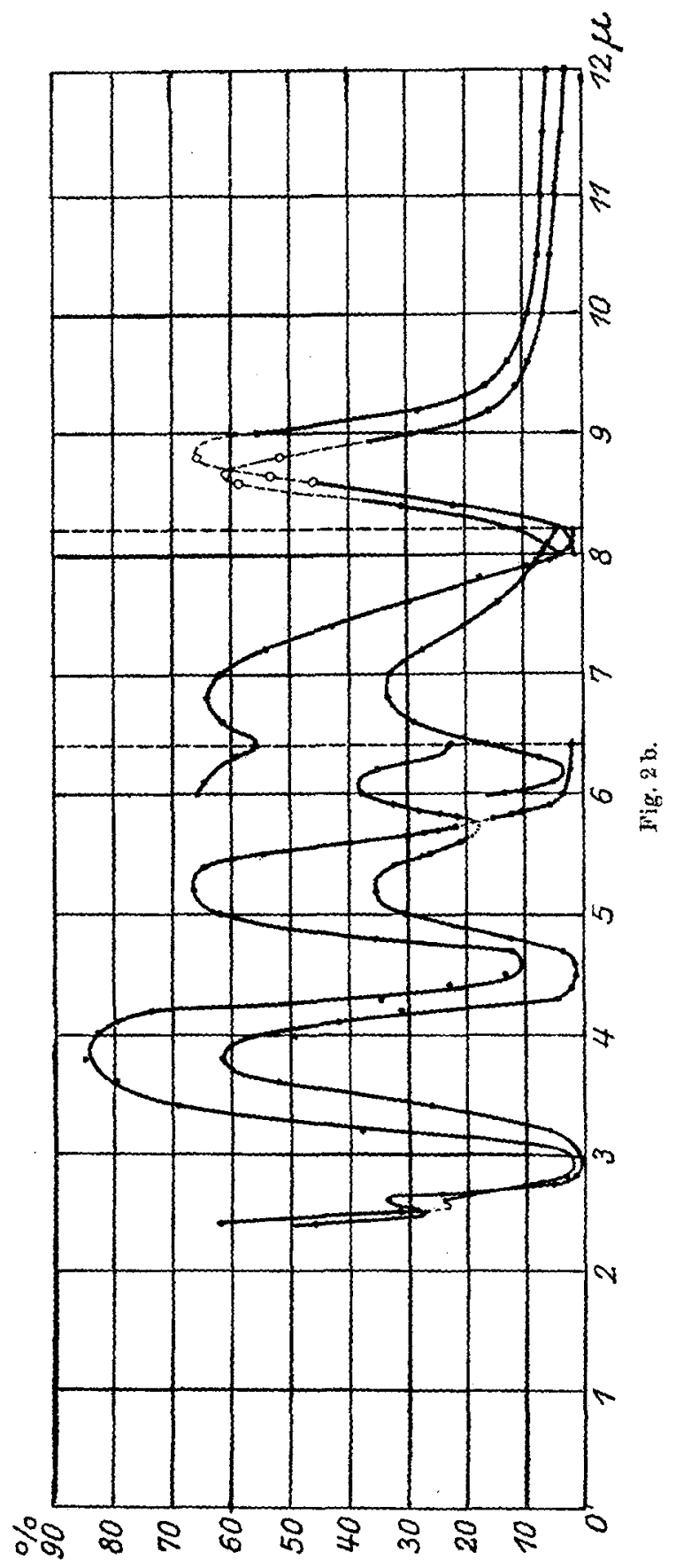

Zeitsehrift fur Physik. Bd. Vt. 
der höher liegenden Kurve entspricht möglicherweise eine etwas langwelligere in der anderen.

Der Verlauf der Achsendispersion (Fig. 2a) ist für diejenige Symmetrieachse dargestellt, welche für die erste untersuchte Wellenlänge die Achse maximaler Durchlässigkeit ist. Die Kurve zeigt $z$ wischen $3 \mu$ und $4 \mu$ einen schwachen, fast linearen Abfall, anschließend beschreibt sie bis $4,9 \mu$ eine flache, aber deutlich ausgeprägte „Wellenbewegung", die fast genau in das Gebiet der $\mathrm{SO}_{4}$-Bande fällt. Ihre Gesamthöhe beträgt $\sim 4^{0}$, ihr Verlanf wäre vielleicht bei Anwendung engerer Spalten noch ausgeprägter gewesen, dies war aber bei der starken Absorption nicht möglich. Eine Ähnlichkeit mit der Achsenbewegung, wie sie von Herrn $D$ afet ${ }^{1}$ ) am Neodymsulfat innerhalb der Absorptionsbande im Orange gefunden wurde, und dem Verlauf des Brechungsindex bei anomaler Dispersion ist danach unverkennbar. Nach einem schwachen Maximum bei $\sim 5 \mu$ krümmt sich die Kurve rasch gegen die Abszissenachse mit zunehmender Annäherung an die Wellenlänge $5,76 \mu$.

Der Verlauf der Achsendrehung in der unmittelbaren Umgebung dieses Punktes ist nicht mit Sicherbeit eindeutig zu bestimmen. Nimmt man an, wie dies in Fig. $2 \mathrm{~b}$ gezeichnet ist, daß bei 5,76 $\mu$ ein Schnittliankt der beiden Hauptdurchlässigkeitskurven vorhanden ist, so folgt daraus der in Fig. 2 a wiedergegebene Verlauf der Achsendrebung. Das Vorhandensein des Schnittpunktes bedingt nämlich, daß der Achse maximaler Durchlässigkeit für die letzte vor dem Schnittpunkt untersuchte Wellenlänge der Achse minimaler Durchlässigkeit für die erste dahinterliegende entsprechen muß. Es ist jedoch noch eine zweite Deutung des Verlaufs der Achsendrehung möglich, wenn man nämlich annimmt, daß die beiden Hauptdurchlässigkeitskurven sich nicht schneiden, sondern daß die $D_{\max }$-Kurve bei $5,76 \mu$ lediglich eine Einsenkung besitzt, welche bis dicht an die $D_{\min }$-Kurve heranreicht. In diesem Falle wird der Gang dor Achsendrehang in Fig. 2 a durch die panktierte Linie dargestellt. Nach dem beobachteten Verlauf der Hauptdurchlässigkeitskurven ist allerdings die Annahme eines Schnittpunktes die bei weitem wahrscheinlichere.

Wegen der wachsenden Abnahme der Durchlässigkeit mit zunehmender Wellenlänge wurden die Messungen mit dem dickeren Gipsplättchen bei $6,4 \mu$ abgebrochen, der Versuch, sie noch von $3 \mu$ $\mathrm{ab}$ in Richtung kürzerer Wellen fortzasetzen, gelang bei dem geringen Dichroismus nur im beschränkten Maße. Soweit die Messungen

1) H. Dufet, 1. c. 
Über die Dispersion der optischen Symmetrieachsen von Gips, Augit usw. 19

reichen, scheinen die Symmetrieachsen wieder eine wellenartige Bewegung mit dem Zentrum bei $2,65 \mu$, ähnlich der bei $4,4 \mu$ gelegenen, auszuführen. Bemerkenswert ist, daß sich aus den aufeinanderfolgenden Drehkurven für $2,5 \mu, 2,6 \mu, 2,65 \mu$ und $2,75 \mu$ sicher auf das Vorliegen von zwei Schnittpunkten der Hauptdurchlässigkeitskurven bei etwa 2,52 $\mu$ und 2,70 $\mu$ schließen läßt. Denn da sich die Lagen der Achsen kleinster Durchlässigkeit für $2,60 \mu$ und $2,65 \mu$ stetig an die Lagen der Achsen größter Durchlässigkeit für die vorangehenden and folgenden Wellenlängen $(2,5 \mu$ bzw. $2,75 \mu)$ anschließen (vgl. Tab. 1), müssen sowohl zwischen $2,5 \mu$ und $2,6 \mu$ als auch zwischen $2,65 \mu$ und $2,75 \mu$

Tabelle 1. Gips $\left(\mathrm{CaSO}_{4} \cdot 2 \mathrm{H}_{2} \mathrm{O}\right)$.

Bezugsebene: (0+10).

Bezugsrichtung: faseriger Bruch.

\begin{tabular}{|c|c|c|c|c|c|c|c|c|c|c|c|}
\hline$\lambda$ & $\begin{array}{c}D_{\text {max }} \\
\text { Proz. }\end{array}$ & $\begin{array}{c}D_{\min } \\
P_{\text {roz. }}\end{array}$ & $\begin{array}{c}P_{\max } \\
0 \\
\end{array}$ & $\begin{array}{c}P_{\min } \\
0 \\
\end{array}$ & $\begin{array}{c}\varphi_{0} \\
0 \\
\end{array}$ & $\lambda$ & $\begin{array}{c}D_{\text {max }} \\
\text { Proz. }_{1}\end{array}$ & $\begin{array}{c}D_{\min } \\
\text { Proz. }\end{array}$ & $\begin{array}{c}P_{\max } \\
0 \\
\end{array}$ & $\begin{array}{c}\varphi_{\min } \\
0\end{array}$ & $\begin{array}{c}\varphi_{0} \\
0 \\
\end{array}$ \\
\hline & \multicolumn{5}{|c|}{ Durchlässigkeit, $d=0,15_{6} \mathrm{~mm}$} & & \multicolumn{5}{|c|}{ Durchlässigkeit, $d=0,04_{3} \mathrm{~mm}$. } \\
\hline $\begin{array}{l}2,4 \\
2,5\end{array}$ & 61,9 & $\begin{array}{l}45,8 \\
27,4\end{array}$ & $\begin{array}{l}113,4 \\
115,4\end{array}$ & $\begin{array}{l}23,4 \\
25,4\end{array}$ & $\begin{array}{l}113,4 \\
115,4\end{array}$ & $\begin{array}{l}6,0 \\
6,1\end{array}$ & 65,4 & $\begin{array}{r}13,7 \\
5,1\end{array}$ & - & $\overline{-}$ & $\overline{-}$ \\
\hline 2,6 & & 23,4 & 22,5 & 112,5 & 2,5 & 6,3 & 3 & 7,7 & 114,3 & 24,3 & 24,3 \\
\hline 2,65 & & 19,8 & 19,6 & 109,6 & 09,6 & 6,4 & & 16,4 & 13,0 & 23,0 & 23,0 \\
\hline 2,75 & & 0 & 105,5 & 15,5 & 05,5 & 6,6 & & 9,1 & 12,7 & 22,7 & 22,7 \\
\hline 2,8 & & & 7,8 & 8 & 07,8 & 6,8 & & 3,3 & 3,0 & 23,0 & 23,0 \\
\hline 2,9 & & 1,1 & 7 & 7 & 8 & 7,0 & & 2 & 3,0 & 23,0 & 23,0 \\
\hline 3,0 & & & 66,9 & 9 & 06,9 & 7,2 & & 4 & 4,8 & 24,8 & 24,8 \\
\hline 3,2 & & & 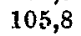 & & 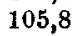 & 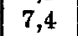 & & & & 25,4 & 25,4 \\
\hline 3, & & 26 & 7 & 7 & 10 & 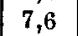 & & & & 21,8 & 21 , \\
\hline 0 & & & 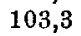 & & & & & & & 11,1 & 11,1 \\
\hline 3,8 & & & 102 & 4 & 102 & 7 , & & 3 & & 168 & -12 \\
\hline 4,0 & & & & & & & & & & 15 & 25 \\
\hline 4,1 & & & $\mathbf{1}$ & 8 & & 8 & 7, & 3 & 21 & 11 & 21 \\
\hline 4,2 & & & & & & & 6 , & 2 & $z$ & 11 & 21 \\
\hline $\mathbf{4 , 4}$ & & & 1 & 3 & 10 & 8, & 5 , & 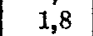 & 24 & 114 & 24 \\
\hline 4,5 & & 1, & 100,8 & 10,8 & 100,8 & & & & 22 & 112 & סוג \\
\hline 4,6 & & 1 & 98 & 9,9 & & & \multicolumn{5}{|c|}{ Reflexion } \\
\hline 4,7 & 1 & 3 , & 100,4 & 10,4 & $10 c$ & & & & & & \\
\hline$\therefore$ & & 12 & & & & & $\kappa_{\max }$ & $\min$ & & & \\
\hline 5,0 & & & 1 & 5 & 101,5 & 8,0 & 4,0 & 1,4 & - & - & - \\
\hline 5,2 & & & 11 & & 100,4 & 8,2 & & 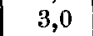 & 110,9 & 20,9 & 20 \\
\hline 5,4 & & & 97 & 7 & & 8,4 & & 22,1 & 110,0 & 20 , & \\
\hline 5 & & & & & & 8 & $x=1$ & $(45$ & & & \\
\hline 5,6 & & & 9 & & & 8,65 & $(61,0)$ & $(53,2)$ & \multicolumn{3}{|c|}{ Deformiert } \\
\hline 5,6 & & & & & & 8 , & 8) & $(51,8)$ & & & \\
\hline 5, & & & 80 & 1 & & 9 , & 55,4 & 29,3 & $\tau, 6$ & 97,6 & \\
\hline & & & & & & 9 , & & & 11,7 & & 14 \\
\hline 5 & & & 7 & 16 & & 9 & & 11 & 13,9 & 103,9 & 13 \\
\hline 5 & & & & & & 9 & 12, & & 15,1 & 105,1 & 15 \\
\hline 5 & & & & & & 10, & 9 , & 6 & 19,0 & & \\
\hline 5,8 & & & & & & & 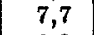 & 5, & 24 & & \\
\hline 8 & & 5 & & & & & 6 & 4 & 28,0 & $118_{30}$ & 0 \\
\hline 6, & & & & & & & & $\delta_{2}$ & & & 28 \\
\hline 6,2 & & 2, & & & 25,4 & 12,0 & 5,8 & 3,1 & 26 & 116 & 26 \\
\hline 6,4 & & 2 & & & & & & & & & \\
\hline
\end{tabular}


die Achsen größter und kleinster Durchlässigkeit ihre Rolle tauschen, d. h. sich die Hauptdurchlässigkeitskurven schneiden. Indem hierdurch die Möglichkeit von Schnittpunkten der Hauptdurchlässigkeitskurven dargetan wird, erhält die oben diskutierte Annahme eines solchen bei $5,76 \mu$ eine Stütze.

Für das sichtbare Gebiet sind einige Werte nach den Berekschen Messungen ${ }^{1}$ ) eingetragen, die ein flaches Maximum bei $\sim 0,57 \mu$ ergeben. Bemerkenswert ist die große Abweichung (minimal 380) von den letzten Werten der vorliegenden Messungen, es müssen also in dem dazwischenliegenden Gebiet noch erhebliche Drehungen stattfinden.

Für die Untersuchung des Gebietes von 6,4 $\mu$ bis an die Grenze der metallischen Reflexion wurde das Gipsplättchen von $0,04_{\mathrm{g}} \mathrm{mm}$ Dicke verwandt. Zur Kontrolle wurde auch bei diesem die Lage der Symmetrieachsen für $6,4 \mu$ ermittelt, sie stimmt bis auf $0,2^{\circ}$ mit der für das dickere Spaltstück erhaltenen überein, also völlig innerhálb der Versuchsfehler. Ebenso fügt sich der übergreifend bestimmte Wert für $6,3 \mu$ gut in die Dispersionskurve der Symmetrieachsen ein. Zur besseren Deutung des Verlaufs der Hauptdurchlässigkeitswerte wurden diese noch allein für $6,0 \mu$ und $6,1 \mu$ unter Zugrundelegung der bereits bekannten Achsenlagen ermittelt. Hiernach zeigt die $D_{\min }$-Kurve eine starke, relativ scharfe Bande bei $6,2 \mu$, die $D_{\text {max }}$-Kurve eine schwache bei $6,4 \mu$, weiterhin fallen beide Kurven nach einem Gebiet etwas größerer Durchlässigkeit rasch ab. Über $8,2 \mu$ hinaus ließen sich die Messungen nicht vortreiben, doch genügte es, um an die darauf folgenden Reflexionsmessungen Anschluß zu erhalten.

Der Verlauf der Dispersion der Symmetrieachsen zeigt ein flaches Minimum bei $6,6 \mu$, darauf einen leichten Anstieg, der hinter $7,4 \mu$ in einen rapiden Abfall übergeht. Die letzten aufgenommenen Punkte $(7,95 \mu$ bis $8,2 \mu)$ sind infolge der nur wenige Millimeter betragenden Ausschläge für die durchgelassene Energie ziemlich gestreut, sie lassen indes auf einen Abfall mit wachsender Wellenlänge schließen. Ganz ansicher ist der Punkt 7,9 $\mu$, für den der Dichroismus fast verschwindet. Eine nähere Untersuchung war bei den ungünstigen Meßverhältnissen aussichtslos. Die Anordnung des hinter 7,92 $\mu$ liegenden Kurventeiles entspricht der angenommenen Überschneidung der Hauptdurchlässigkeitskurven bei $7,92 \mu$ and der damit verbundenen Uberführung der $D_{\min }$-Achse in die $D_{\text {max }}$-Achse. Auch hier ist ebenso wie bei $5,76 \mu$ eine zweite Art der Darstellung möglich, wenn man annimmt, daß

1) AT. Berek, 1. c. 
die Hauptdurchlässigkeitskurven sich n i c b t schneiden, letztere Möglichkeit ist aber wiederum wenig wahrscheinlich.

Die Untersuchungen warden dann mit Hilfe von Reflexionsmessungen an einer natürlichen Spaltfläche, und zwar der (010)-Ebene, einer $\sim 9 \times 11 \mathrm{~cm}$ großen Gipsplatte aus demselben Material fortgesetzt. Bezugsrichtung war wieder die Richtung des faserigen Bruches, so $\mathrm{dab}$ die Resultate an die bisherigen ohne weiteres anschlossen. Die optische Beschaffenheit des benntzten Flächenstïcks war befriedigend, wenn auch nicht von der Güte geschliffener Flächen. Zur Kontrolle wurde zunächst die Lage der Symmetrieachsen für $8,2 \mu$ bestimmt, die Abweichung von dem ans den Durchlässigkeitsmessungen abgeleiteten Wert beträgt $\sim 1^{0}$ und liegt innerhalb der Fehlergrenze. Dabei entspricht der Achse maximaler Durchlässigkeit die Achse minimaler Reflexion. Für $8,0 \mu$ wurden nur $R_{\max }$ und $R_{\min }$ zur Vervollständigung der Hauptreflexionskurven bestimmt.

Letztere bestehen aus zwei scharfen, etwas gegeneinander verschobenen Erhebungen im Gebiet von $8 \mu$ bis $9,5 \mu$, die sich offenbar bei etwa $8,7 \mu$ schneiden. Genauere Angaben bezüglich der Umgebung von $8,7 \mu$ werden durch das Auftreten systematischer Verzerrungen der Drehkarven für $8,6 \mu, 8,65 \mu$ und $8,8 \mu$ verhindert. Demzufolge stellen die Maximal - and Minimalwerte dieser Kurven (in Fig. 2b umringelt eingetragen) nur annähernd die Hauptreflexionswerte dar, für die Ableitung der Achsenlagen sind sie leider völlig unbraucbbar. In Fig. 4 ist als Probe eine derartige Anfeinanderfolge verzerrter Drehkurven $(10,3 \mu, 10,4 \mu, 10,6 \mu)$ für den noch zu besprechenden Augit wiedergegeben, die deformierten Kurven für Gips $8,6 \mu, 8,65 \mu, 8,8 \mu$ gleichen in der Form ungefähr den Spiegelbildern der erstgenannten an der Ordinatenachse. Die Entstehung der Verzerrungen erklärt sich aus der spektralen Unreinheit der Spektrometerstrahlnng, indem in den Wellenlängenbereichen, in denen die Verzerrungen auftreten, die Komponenten des Strahlungsgemisches erbeblich voneinander abweichende Drehkurven liefern, aus deren Utberlagerung die deformierten Kurven hervorgehen.

Für $8,2 \mu$ und $8,4 \mu$ und dann wieder von $9 \mu \mathrm{ab}$ war die Lage der Symmetrieachsen in gewöhnlicher Weise bestimmbar: die ersten beiden Werte geben einen schwachen Abfall der Dispersionskurve, von $9 \mu \mathrm{ab}$ hat man einen relativ steilen, anfangs gekrümmten Anstieg, der zwischen $9,5 \mu$ und $11 \mu$ in einen fast linear verlaufenden Teil übergeht. Die letzten beiden Werte $(11,5 \mu$ und $12 \mu)$, die aus ziemlich mangelhaften Drehkurven abgeleitet sind, können lediglich beweisen, $\mathrm{da} \beta$ sich der lineare Anstieg nicht fortsetzt. Uberhaupt ist bei dem 
weiteren Abstand der letzten Punkte die Kurve möglicherweise komplizierter, für eingehendere Messungen sind aber die Versuchsbedingungen zu ungünstig. Die gegenseitige Orientierung der Kurvenzweige beiderseits $8,7 \mu$ entspricht der angenommenen Durchschneidnng der Hauptreflexionskurven in diesem Punkt.

Augit $\left[\mathrm{Ca}(\mathrm{Fe}, \mathrm{Mg}) \mathrm{Si}_{2} \mathrm{O}_{6}\right]$. Uber die Dispersion der optischen Symmetrieachsen im Augit liegen im sichtbaren Spektralgebiet Messangen von Herrn Wülfing ${ }^{1}$ ) vor.

Tabelle 2. Augit $\left[\mathrm{Ca}(\mathrm{Fe}, \mathrm{Mg}) \mathrm{Si}_{2} \mathrm{O}_{6}\right]$.

Bezugsebene: $(\overline{0} \overline{10})$.

Bezugsrichtung: Vertikalachse.

\begin{tabular}{|c|c|c|c|c|c|c|c|c|c|c|c|}
\hline $\begin{array}{l}\lambda \\
\mu \\
\end{array}$ & $\begin{array}{r}R_{\max } \\
\text { Prox. }\end{array}$ & $\begin{array}{r}R_{\text {min }} \\
\text { Proz. }\end{array}$ & $\begin{array}{c}\varphi_{\max } \\
0 \\
\end{array}$ & $\begin{array}{c}\varphi_{\min } \\
0 \\
\end{array}$ & $\begin{array}{c}\varphi_{0} \\
\mathbf{0} \\
\end{array}$ & $\lambda$ & $\begin{array}{l}R_{\max } \\
\text { Proz. } \\
\end{array}$ & $\begin{array}{c}R_{\min } \\
\text { Proz. } \\
\end{array}$ & $\begin{array}{c}\varphi_{\max } \\
0 \\
\end{array}$ & $\begin{array}{c}\varphi_{\min } \\
0 \\
\end{array}$ & $\begin{array}{r}\varphi_{0} \\
0 \\
\end{array}$ \\
\hline 8,6 & $(6,8)$ & $2,0)$ & \multicolumn{3}{|c|}{ Deformiert } & 10,4 & $(49,2)$ & \multirow{2}{*}{$\begin{array}{l}(34,2) \\
(30,9)\end{array}$} & \multirow{2}{*}{\multicolumn{3}{|c|}{ miert }} \\
\hline 8,8 & 21,4 & 3,5 & 100,8 & 10,8 & 10,8 & 10,6 & $(63,0)$ & & & & \\
\hline 9,0 & 57,0 & 6,7 & 100,6 & 10,6 & 10,6 & $10 ; 8$ & 68,9 & 25,0 & 0,5 & 90,5 & 90,5 \\
\hline 9,2 & 69,0 & 7,4 & 101,0 & 11,0 & 11,0 & 11,0 & 69,8 & 21,8 & 0,7 & 90,7 & 90,7 \\
\hline 9,4 & 62,3 & 7,0 & 101,9 & 11,9 & 11,9 & 11,2 & 70,8 & 19,8 & 0,9 & 90,9 & 90,9 \\
\hline 9,6 & 41,3 & 6,3 & 102,6 & 12,6 & 12,6 & 11,4 & 68,7 & 18,3 & 1,4 & 91,4 & 91,4 \\
\hline 9,7 & $(26,7)$ & $7,7)$ & & & & 11,6 & 59,7 & 16,2 & 1,6 & 91,6 & 91,6 \\
\hline 9,8 & $(22,6)$ & $(11,2)$ & Defo & miert & & 11,8 & 41,6 & 14,3 & $\mathbf{2 , 0}$ & 92,0 & 92,0 \\
\hline 9,9 & $(29,0)$ & $(13,9)$ & & & & 12,0 & 26,9 & 12,9 & 2,5 & 92,5 & 92,5 \\
\hline 10,0 & 38,0 & 12,7 & 58,3 & 148,3 & 58,3 & 12,2 & 21,0 & 11,5 & 2,5 & 92,5 & 92,5 \\
\hline 10,1 & 46,5 & 12,2 & 63,9 & 153,9 & 63,9 & 12,5 & 16,4 & 10,7 & 2,7 & 92,7 & 92,7 \\
\hline 10 & 48, & 15 & 68,9 & 158,9 & 68,9 & 12,8 & 13,2 & 9,6 & 1,1 & 91,1 & 91,1 \\
\hline & $10^{\circ}$ & ( & & & & & & & & & \\
\hline
\end{tabular}

Das bei der vorliegenden Untersuchung benutzte Material bestand in einem $0,46 \mathrm{~mm}$ dicken Dünnschliff und einer $5,4 \times 5,8 \mathrm{~cm}$ großen, aus zwei gleichen Schnittstücken zusammengesetzten Fläche aus einem aus Nordmarken (Schweden) stammenden Kristall. Als Bezugsrichtung diente die Richtung der Vertikalachse, deren Lage durch zahlreiche feine Sprünge gekennzeichnet war. Bei den Reflexionsmessungen war die reflektierende Oberfläche die (0) $\overrightarrow{0})$-Ebene. Da der positive.Drehungssinn des Kristalls beibehalten wurde, ergeben sich die Winkelwerte der Achsenlagen für die (010)-Ebene durch Umkehr der Vorzeichen.

Der Dünnschliff erwies sich nach orientierenden Messungen im Gebiet von $1 \mu$ bis $8 \mu$ für den vorliegenden $Z_{\text {weck }}$ als ungeeignet, da entweder der Dichroismus zu schwach oder die Absorption za groß war. Ebenso versagte hier die Reflexionsmethode wegen der geringen Verschiedenheit der Reflexionsvermögen. Da, soweit festgestellt, überhanpt noch keine Durchlässigkeits- und Reflexionsmessungen für Augit im kurzwelligen Ultrarot vorliegen, wurden aus

3) Entnommen aus H. Dufet, Recueil des donnees numeriques, T. II. 
Über die Dispersion der optischen Symmetrieachsen von Gips, Augit usw. 23

den Meßreihen dureh Mittelbildung zwischen den Durchlässigkeitsbzw. Reflexionswerten zweier um $90^{\circ}$ verschiedenen Lagen die entsprechenden Werte für natürliche Strablung errechnet. Sie sind in Fig. 3 dargestellt, tragen indes nur orientierenden Charakter.

Bessere Ergebnisse wurden in dem ziemlich ausgedehnten Gebiet selektiver Reflexion zwischen $8,5 \mu$ und $13 \mu$ erzielt (Tab. 2, Fig. 5 a und b). Wie beim Gips treten in einzelnen Spektralgebieten Verzerrangen in den

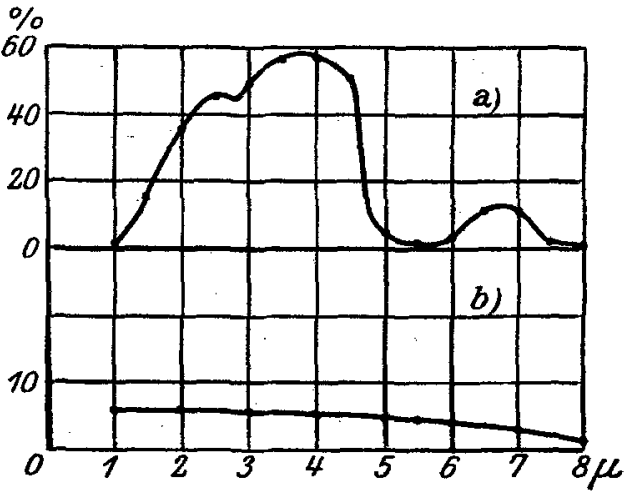

Fig. 3. $a$ Durchlässigkeit, $b$ Reflexionsvermögen Drehkurven auf, und zwar zwischen $9,7 \mu$ bis $9,9 \mu, 10,3 \mu$ bis $10,6 \mu$ (in Fig. 4 dargestellt) und für die erste untersuchte Wellenlänge 8,6 $\mu$. Der Vergleich der deformierten Kurven untereinander läßt weiter gewisse Beziehungen erkennen, z. B.

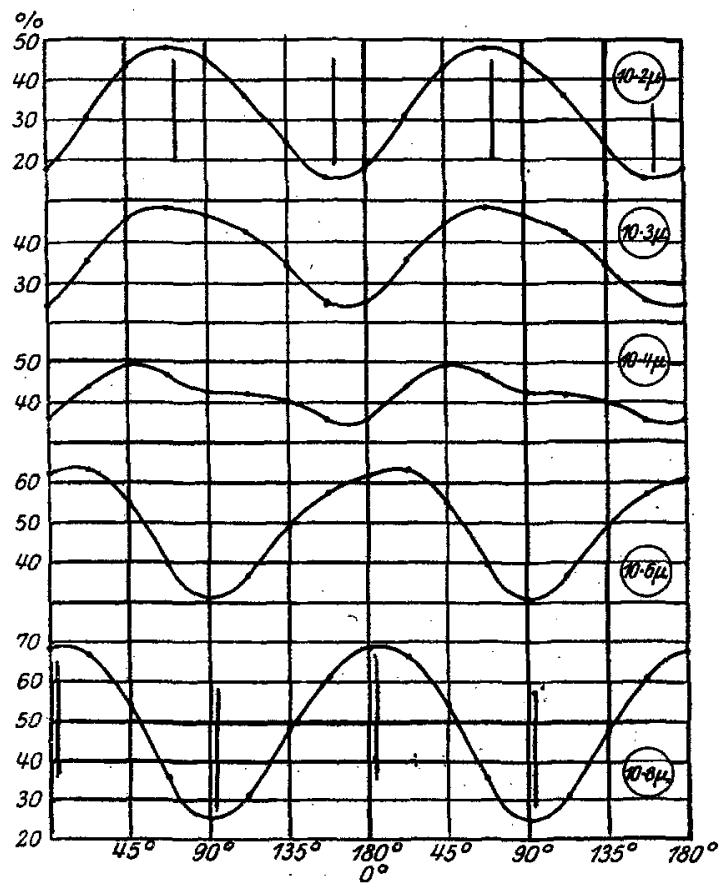

Fig. 4. 
ähnelt die Drehkurve für $9,7 \mu$ der für $10,3 \mu$, ferner die Kurve $9,9 \mu$ der Kurve $10,6 \mu$, wenn man sich letztere nacheinander an der Ordinatenand Abszissenachse gespiegelt denkt. Die Lage der Symmetrieachsen und

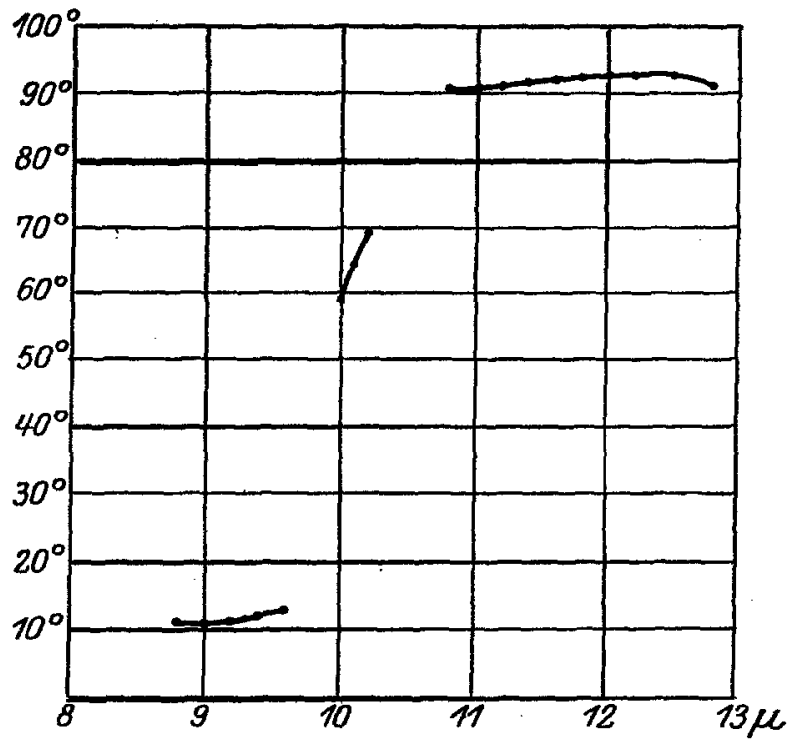

Fig. 5 a.

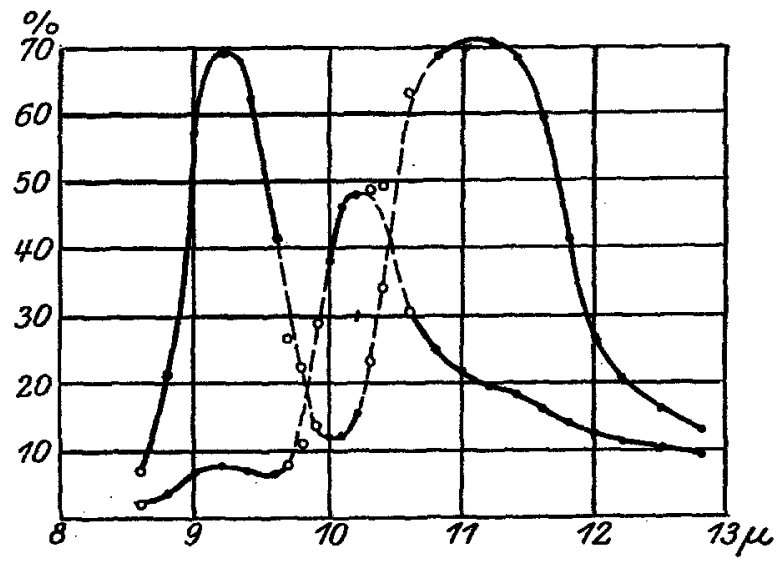

Fig. 5 b.

die Hauptreflexionswerte bleiben in den betreffenden Spektralgebieten unbestimmbar ${ }^{1}$ ), bezüglich des Verlaufs der letzteren ergibt sich die in Fig. $5 \mathrm{~b}$ dargestellte Form mit den beiden Schnittpunkten bei $9,8^{r} \mu$

1) Die Extremwerte der verzerrten Kurven sind in Fig. $5 \mathrm{~b}$ wieder umringelt eingetragen. 
und $10,45 \mu$ als die natürlichste. Die eine der beiden Reflexionskurven besteht danach in einer schmaleren und einer breiteren starken Erhebung bei $9,25 \mu$ bzw. $11,1 \mu$, die durch eine tiefe Einsenkung bei $10,05 \mu$ voneinander getrennt sind, die andere in einer schwächeren Erhebung bei $10,25 \mu$ und einer sehr flachen bei $\sim 9,2 \mu$. Der zam Teil enorme Unterschied der Hauptreflexionswerte begünstigte die Genauigkeit der Achsenbestimmung. Der Gang der Achsendispersion ist in Fig. 5a für diejenige Symmetrieachse dargestellt, welche zu Anfang die Achse minimaler Reflexion bildet. Die Kurve zeigt zwischen $8,8 \mu$ und $9,6 \mu$ einen schwachen Anstieg von kaum $3^{0}$. Für $9,7 \mu, 9,8 \mu$ und $9,9 \mu$, der Umgebung des ersten Schnittpunktes, sind, wie erwähnt, die Drehkurven verzerrt. Die Lage des Punktes $10,0 \mu$ ist gegen die des Punktes $9,6 \mu$ um fast genau $45^{\circ}$ verschoben, und zwar unter Berücksichtigung des Schnittpunktes der Hauptreflexionskurven im Sinne wachsender Winkel. Er bildet zusammen mit $10,1 \mu$ und $10,2 \mu$ einen steil ansteigenden, annäherud livearen Kurventeil. Die folgenden, bei $10,3 \mu, \cdot 10,4 \mu$ und $10,6 \mu$ ausgeführten Messungen müssen wegen starker Deformation der Drehkurven wieder ausscheiden. Von $10,8 \mu$, dessen zugehöriger Winkelwert auf Grund der erneuten Überschneidung um $\sim 20^{\circ}$ größer als der bei $10,2 \mu$ anzunehmen ist, bis $12,8 \mu$, der letzten untersachten Wellenlänge, ist die Achsendrehung wie anfangs sehr gering; sie zeigt ein schwaches, fast lineares Anwachsen, ohne irgendwelche erkennbare Anomalien und ein flaches Maximum bei $\sim 12,5 \mu$.

Adular $\left[(\mathrm{K}, \mathrm{Na}) \mathrm{AlSi}_{3} \mathrm{O}_{8}\right]$. Für das sichtbare Gebiet ist die Dispersion der optischen Symmetrieachsen von Herrn Petrow ${ }^{1}$ ) bestimmt worden.

Tabelle 3. Adular $\left[\left(\mathrm{K}_{1} \mathrm{Na}\right) \mathrm{AlSi}_{3} \mathrm{O}_{8}\right]$.

Bezugsebene: (0 $\overline{1} 0)$, Bezugsrichtung: Klinoachse.

\begin{tabular}{|c|c|c|c|c|c|}
\hline $\begin{array}{r}\lambda \\
\mu\end{array}$ & $\begin{array}{c}R_{\max } \\
\text { Proz. }\end{array}$ & $\begin{array}{c}R_{\text {min }} \\
\text { Proz. }\end{array}$ & $\begin{array}{c}\varphi_{\max } \\
0\end{array}$ & $\varphi_{\min }$ & $\begin{array}{c}\varphi_{0} \\
0\end{array}$ \\
\hline 8,2 & 8,2 & 4,3 & 168 & 78 & -12 \\
\hline 8,4 & 29,1 & 17,0 & 175 & 85 & -5 \\
\hline 8,6 & 48,5 & 36,4 & 6 & 96 & +6 \\
\hline 8,8 & 47,3 & 36,7 & 22 & 112 & 22 \\
\hline 9,0 & 38,8 & 25,7 & 37 & 127 & 37 \\
\hline 9,05 & $35, \tilde{5}$ & 26,1 & 45 & 135 & 45 \\
\hline 9,1 & 35,4 & 27,0 & 66 & 156 & $66(-24)$ \\
\hline 9,2 & 42,5 & 23,1 & 94 & 4 & $94(+4)$ \\
\hline 9,4 & 55,3 & 20,3 & 102 & 12 & 102 \\
\hline 9,6 & 51,0 & 29,0 & 98 & 8 & 98 \\
\hline 9,8 & 38,6 & 29,1 & 105 & 15 & 105 \\
\hline 10,0 & 29,6 & 24,6 & 133 & 45 & $(133)($ \\
\hline
\end{tabular}

1) K. Petrow, Neues Jahrb. f. Min., Beil.-Bd. 37, 457, 1914. 


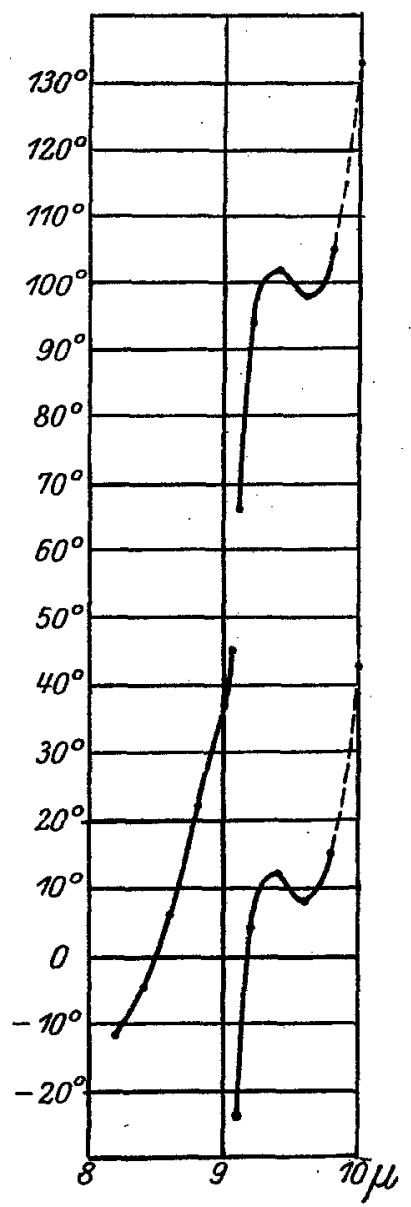

Fig. 6a.

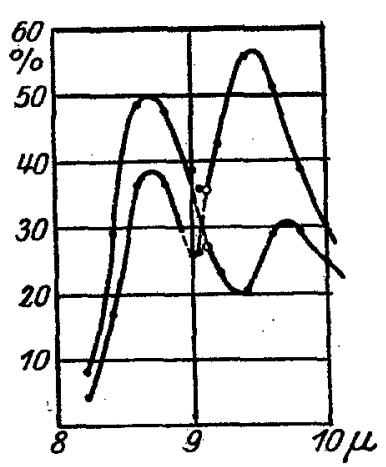

Fig. 6 b.
Die vorliegenden Untersuchungen mußten sich aus den gleichen Gründen wie beim Augit auf das Gebiet selektiver Reflexion $(8 \mu$. bis $10 \mu)$ beschränken. Die benutzte Kristallfläche $(\sim 6 \times 4 \mathrm{~cm})$ war aus gut aneinandergepaßten Schnittstücken eines vom St. Gotthard stammenden Kristalls zusammengesetzt und bildete die (0T0)-Ebene. Als Bezugsrichtung diente die Klinoachse, deren Richtung sich durch feine geradlinige Sprünge kennzeichnete, sie bildet in der (010)-Ebene einen Winkel von $64^{\circ}$ mit der Vertikalachse. Wie beim Angit wurde der positive Drehungssinn beibehalten.

Verzerrte Kurven nach Art der bei Gips und Angit auftretenden zeigen sich hier nicht. Bezüglich des Verlaufs der Hauptreflexionskurven (Fig. 6 b) bleibt es zweifelhaft, ob. in der Umgebung von $9,1 \mu$ ein Schnittpunkt anzunehmen ist, oder ob die höher gelegene Kurve die andere im ganzen untersuchten Spektralgebiet einschließt. In Fig. $6 \mathrm{~b}$ sind beide Möglichkeiten angedeutet. Im übrigen besitzen die Hauptreflexionskurven je zwei Maxima, eins gemeinsam bei $8,7 \mu$ und je ein weiteres bei $9,5 \mu$ und $9,7 \mu$. Die Dispersion der optischen Symmetrieachsen ist in Fig. $6 \mathrm{a}$ zu Beginn für die $\boldsymbol{R}_{\max }$-Achse dargestellt. Im Gegensatz zum Augit zeigt die Kurve bereits vom Rande des selektiven Reflexionsgebietes an einen sebr steilen Anstieg bis hinter $9 \mu$. Der Annahme eines Schnittpunktes der Hauptreflexions. kurven entspricht dann eine weitere Fortsetzung der bisherigen Bewegung bis $\sim 9,2 \mu$, an die sich nach einer vorübergehenden Umkehr eine erneute Achsendrehung im Sinne wachsender Winkel anzuschließen scheint. Im anderen Falle 
wäre der Kurventeil von $9,1 \mu$ ab um $90^{\circ}$ tiefer za legen (vergleiche Fig. 6a).

\section{MeBgenauigkeit.}

Bezüglich der Genauigkeit in der Bestimmung der Lage der Symmetrieachsen ist wesentlich, daß alle Fehler, die im Verwendungsbereich eines Präparates additive Konstanten sind (z. B. Orientierungsfehler des Kristalls gegen die Indexlinie infolge unscharfer äußerer Kennzeichnung der Vorzugsrichtangen, Betrag schätzungsweise \pm 1 bis $2^{\circ}$ ), zwar den Absolutwert der Achsenlagen, nicht aber den Gang der Achsendispersion, auf den es hauptsächlich ankommt, beeinflussen. Die Unsicherheit der "relativen" Lagen der Symmetriachsen (d. h. bezogen auf die Nachbarwerte) beträgt bei den Durchlässigkeitsmessungen, nach gelegentlichen Doppelmessungen zu urteilen, nicht mehr als 10, bei den Reflexionsmessungen an Gips und Adular mag sie etwas größer sein.

\section{Schlußbemerkungen.}

Im ganzen lehrt der experimentelle Befund, daß die Stellen stärkster Drehnng der optischen Symmetrieachsen Schnittpunkten der Hauptdurchlässigkeits- bzw. Reflexionskurven sehr nahe liegen 1). Ein Zusammenhang der Achsendrehungen mit den Stellen stärkster Absorption und Reflexion ist im allgemeinen nicht erkennbar. Dies beweist aber keineswegs, daß ein derartiger Einfluß nicht besteht. Vielmehr wird man annehmen müssen, daß bei der Wirkung eines Absorptionsstreifens auf die Achsendrehung ähnliche spektrale Verschiebungen auftreten, wie bei dem Einfluß eines solchen Streifens auf den Brechungsexponenten oder das Reflexionsvermögen.

Es sei noch erwähnt, daß Herr Nakamura ${ }^{2}$ ) auf Grund der Dispersionstheorie einen Ausdruck für den Winkel der Symmetrieachsen mit einer beliebig gewählten Richtung. im Kristall gegeben hat. Indes ist dieser Ausdruck unter der Voranssetzung der Durchsichtigkeit abgeleitet worden, kann daher für das untersuchte Gebiet genauere Gültigkeit nicht beanspruchen.

\section{Zasammenfassung.}

Es wurde die Dispersion der optischen Symmetrieachsen monokliner Kristalle im kurzwelligen Ultrarot verfolgt.

Die Lage der Achsen wurde nach Angabe von Herrn Rubens aus der Abhängigkeit des Durchlässigkeits- bzw. Reflexionsvermögens

1) Die Reflexionsmessungen an Adular liefern allerdings keine direkte Bestätigung dieser Regel, sprechen aber auch nicht dagegen.

2) 8. Nakamura, Phys. ZS. 6, 172, 1905. 
28 Erich Goens, Über die Dispersion der optischen Symmetrieachsen usw.

für senkrecht einfallende, monochromatische und linear polarisierte Strahlung von der Orientierung der Schwingungsebene des elektrischen Vektors gegen den Kristall ermittelt. Die daraus abgeleiteten Schwingungsrichtungen größter and kleinster Durchlässigkeit bzw. Reflexion bezeichneten die Richtungen der Symmetrieachsen. Die zugehörigen größten und kleinsten Werte des Darchlässigkeits- bzw. Reflexionsvermögens ( Hauptdurchlässigkeits- bzw. Hauptreflexionswerte“) wurden ebenfalls den Messungen entnommen.

Die Messungen erstreckten sich auf $\mathrm{Gips}$ von $2,4 \mu$ bis $12 \mu$, Augit von $8,6 \mu$ bis $12,8 \mu$ und Adular von $8,2 \mu$ bis $10,0 \mu$. Der Gang der Achsendispersion wurde zusammen mit den Hauptdurchlässigkeits- bzw. Reflexionswerten als Funktion der Wellenlänge dargestellt.

Vorstehende Arbeit wurde im Physikalischen Institut der Universität Berlin unter Leitung von Herrn Geh. Rat Rubens angefertigt. Herrn Geh. Rat Rubens, dem ich die Anregung zu dieser Arbeit verdanke, spreche ich für die zuteil gewordene Unterstützung meiuen besonderen Dank aus. 